\title{
Wireless Sensor Node Performance Analysis Under Mac Protocol IEEE 802.15.4
}

\author{
Karim Sidibe, Cheick Tidjane Kone, Tchimou N'takpe, Boko Aka, Michel Babri
}

\begin{abstract}
The long-term use of sensors while guaranteeing good performance is a major challenge for sensor networks. To address this issue, it's important to have a good resources management policy. So it is necessary to find the main factors which affected the behaviour of each sensors inside the networks to. In this paper we proposed an enhanced analytical model of CSMA/CA 802.15.4 standard and we study the performance of the proposed model under the MAC parameters and the density of network to find the keys parameters that impacted the quality of services (QoS) of the network.
\end{abstract}

Keywords: Wireless sensor Network, CSMA/CA 802.15.4.

\section{INTRODUCTION}

The use of sensors over a long period while providing an acceptable quality of services (QoS) level is one of the main issues that has received the most attention from researchers. Indeed, it often seems difficult or even almost impossible to recharge or replace sensor batteries when the deployment of sensors for surveillance takes place in areas hostile to humans[1]. This becomes problematic if the system is required to collect information autonomously and over a long period. For example, in agriculture, it is very often necessary to have data on the plant/soil during the period from one harvest to the next. Then, the fundamental question is: How to adapt the dynamics of the sensor nodes according to the state of the network, while respecting the constraints related to the sensors and application?

In order to respond this question, it is essential to define a good policy for the use of sensor node resources in order to meet application requirements in terms of lifetime and QoS (reliability, delay, throughput, etc.). In order to achieve this aim, it is then necessary to determine the key factors that influence QoS level of the Wireless Sensor Network (WSN), which is the main objective of this paper. Several works[2]-[5] have focused on the analysis of WSN

Revised Manuscript Received on February 05, 2020.

* Correspondence Author

Karim Sidibe, Student, Department of Computer Engineering, University Nangui Abrogoua.

Dr Cheick Tidjane Kone, Assistant Professor, Department of Computer Engineering, Lorraine University, Nancy 1, France.

Dr Tchimou N'takpe, Assistant Professor, Department of Computer Engineering, Ecole Nationale d'Electricité et de Mécanique, Nancy, France.

Pr Aka Boko, Professor, Department of Computer Engineering, University Nangui Abrogoua.

Pr Michel Babri, Professor, Department of Computer and Telecommunications, National Polytechnic Institute Félix Houpouët-Boigny of Yamoussoukro (Côte d'Ivoire).

(c) The Authors. Published by Blue Eyes Intelligence Engineering and Sciences Publication (BEIESP). This is an open access article under the CC BY-NC-ND license (http://creativecommons.org/licenses/by-nc-nd/4.0/) performance in order to improve the QoS of a low rate and low power wireless sensor networks. In this paper we are interested to those relating to the radio module because several studies have shown that among the 3 basic units that are: the acquisition unit, the processing unit,

and the radio unit, the radio module remains the element that consumes most energy because it is responsible of communication channel access procedure for data transmission and reception.

Here we study the impact of MAC layer parameters (macMinBE, macMaxCSMABackoffs, and macMaxFrameRetries) variation on the following QoS factors: energy consumption, reliability, and delay. To do so, we propose a modified analytical model of the channel access mechanism CSMA/CA of the IEEE 802.15.4 standard, which is one of the most widely used protocols for communication within Low-Rate Wireless Personal Area Network (LR-WPAN).

When a node wishes to send packets in the network, it competes with other nodes to access the medium. It uses 2 CCAs to detect the status of the transmission channel. If the channel is free then the node can start transmitting, otherwise, it exponentially increases its window size (using the Binary Exponential Backoff algorithm) and then chooses a random number in $\left[0 ; 2^{\wedge \text { macMinBE}}-1\right]$. This number represents the time that must be elapse before the node makes another attempt to access the channel (Backoff time). The number of channel access attempts for a given packet is of course limited. This procedure can lead to unnecessary energy wasting if the backoff mechanism is not efficient. For example, if it can't distinguish between channel occupation by an acknowledgment packet (ACK) and a data packet (DATA), it degrades the network performance [6]. Also because of the large number of nodes encountered in networks, it is quite possible that some nodes transmitted on the channel at the same time. This has the consequence of increasing the collision rate which leads to a degradation of reliability (increase of packet loss rate). Collisions are also due to the problem of hidden nodes. Sometimes, it happens that nodes that are out of range of each other, transmit to the same destination at the same time. This situation also contributes to packet collisions at the destination node. It is therefore essential for a better evaluation of the CSMA/CA protocol of the IEEE 802.15.4 standard to propose a mechanism that takes into account the impact of these problems. This is the subject of our present study. In order to achieve this, we propose to extend the work carried out in [7] and taken up by Zayani et $\mathrm{Al}[8]$ and Kone et $\mathrm{Al}$ [9] through an analysis of the performances of the IEEE 802.15.4 MAC standard. 
The major contribution of this paper is to propose a model and make a more accurate analysis of the influence of the MAC layer parameters that impact the QoS of WSNs. More precisely the proposed model takes into account the hidden nodes and differentiate channel occupation by an ACK and a DATA.

The rest of this article is organized as follows: in the following section, we have an overview of the CSMA/CA mechanism with the IEEE 802.15.4 standard beacon. In section 3, we will present a literature review of recent work related to the analysis and improvement of IEEE 802.15.4 CSMA/CA in beacon enabled mode. Then we present in section 4 our proposed model, then in section 5 we analyze and discuss the numerical results obtained after the simulation of our model and finally we conclude and give some perspectives.

\section{OVERVIEW OF THE IEEE 802.15.4 CHANNEL ACCESS MECHANISM}

The 802.15.4 standard is a communication protocol defined by the IEEE (Institute of Electrical and Electronics Engineers). It is intended for wireless networks of the LR-WPAN (Low Rate Wireless Personal Area Network) family because of the low power consumption, short-range and low data rate of devices using this protocol. It defines the specifications of the physical layer (PHY) and MAC under layer for devices with a low data rate, low power, and short-range communication. It defines 2 operations modes: the first one is the beacon enabled mode. In this mode, a synchronization beacon is emitted periodically by the coordinator node to the other devices in the network, to synchronize them with him for sending data. This allows the node to be active only during this period and then it goes into sleep mode where power consumption is kept to a minimum. The second one is the non-beacon mode. In this mode, there is no synchronization beacon between the coordinator and the other devices. The coordinator node remains in active state because, at any time, each node can send data to him. This standard also defines 2 types of topologies: star and mesh (P2P). In a star topology, communication is only between the PAN coordinator and the nodes, while in the peer-to-peer topology the devices can communicate directly with each other. We have 16 channels in the 2.4 to $2.4835 \mathrm{GHz}$ frequency band, 10 channels in the 902 to $928 \mathrm{MHz}$ frequency band and 1 channel in the 868 to $868.6 \mathrm{MHz}$ frequency band. The access mechanism used by the devices to access these communication channels is CSMA/CA.

When a node has data to send through the network, it competes with other nodes for accessing the communication channel. It initializes the 2 following variables: Backoff number $(\mathrm{NB}=0)$ and Backoff Exponent $(\mathrm{BE}=\operatorname{macMinBE})$, then it randomly chooses a number in the interval $\left[0 ; 2^{\wedge} \mathrm{BE}\right]$. This number denotes the time which must be elapse before the node goes to active mode to start checking the channel status thanks to the 2 CCAs that it performs. If it finds that the channel is not busy then it starts to transmit its data and at the end of its transmission, it receives an acknowledgment packet (ACK) from the receiver. Otherwise, it increases exponentially its window size (thanks to the Binary exponential backoff algorithm) by increasing the current value of $\mathrm{BE}$ and NB by one unit. And it chooses another number (Backoff time) before trying another attempt to access the channel. The attempts number with the same packet is limited and must not exceed the allowed limit macMaxCSMABackoffs. Once it is greater than macMaxCSMABackoffs then the channel access have failed. In this case, the node removes the packet from its queue (buffer). Fig. 1A illustrates the CSMA/CA mechanism that we have just described.

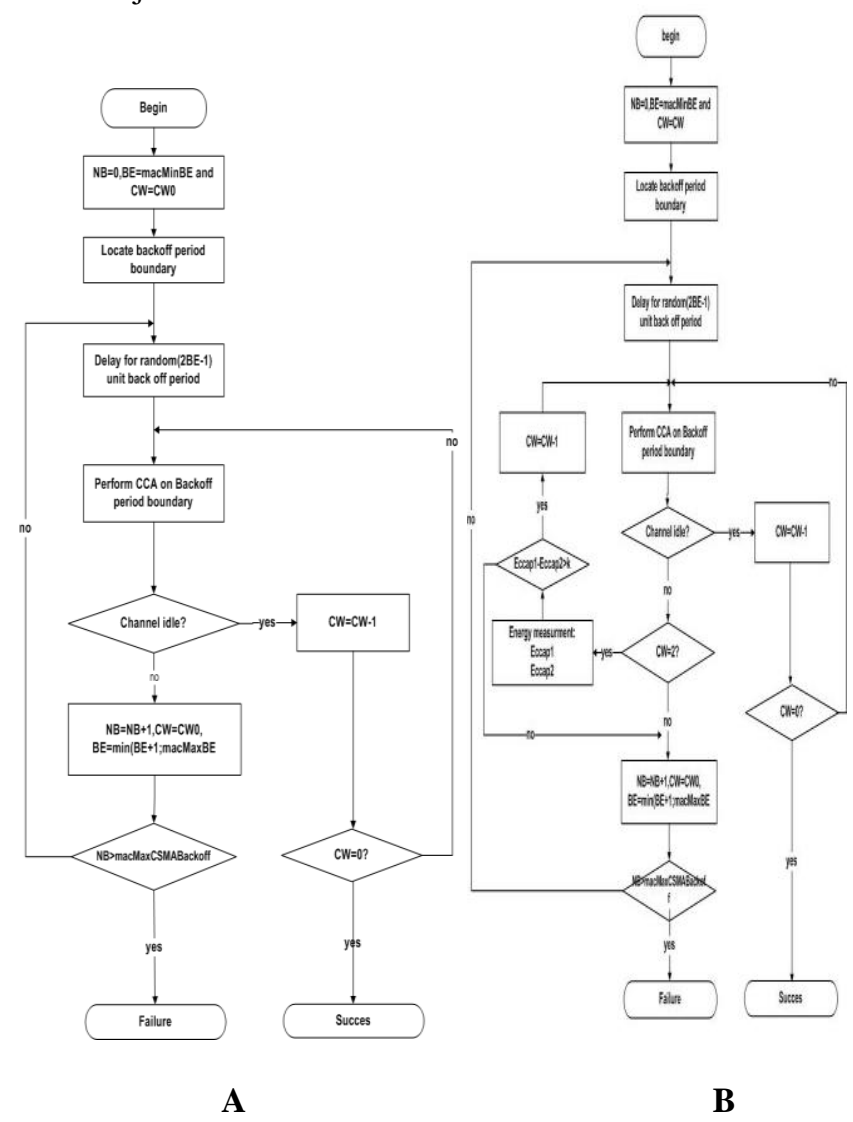

Fig. 1.CSMA/CA IEEE.802.15.4 mechanism

\section{RELATED WORKS}

For better performance management of wireless sensor networks, it is necessary to have a good understanding of the behaviour of each node within the network. Proposing a model that characterizes the performance of the IEEE 802.15.4 MAC standard has been the subject of several major studies. Some of these works are focused on modifying the super frame structure while others relate to the CSMA/CA medium access protocol.

The IEEE 802.15.4 MAC standard with beacon is the most widely used operating mode in LR-WPAN[10]-[12]. The duration of the super frame and the period during which each node competes to access the channel are characterized by the values of the Super frame Order (SO) and Beacon Order (BO). According to the studies in [13], it is shown that SO and $\mathrm{BO}$ are the key parameters to be relied upon to improve the performance of the IEEE 802.15.4 MAC LR-WPAN network. Thus, to ensure a minimum end-to-end delay and low packet losses rate in the network, Khalifeh et Al. (2017)[13] have studied the impact of BO and SO values on network QoS parameters. 
They show that the values of these 2 parameters must be adapted according to the size of the network. For example, in a real-time application, they show that to have an optimal network in terms of delay, packet losses rate, and throughput, one must have $\mathrm{SO}=\mathrm{BO}=3$. In the same way, the authors of [14] shows that it is possible to extend the network lifetime by adjusting the values of both $\mathrm{BO}$ and SO. To do so, they propose a subdivision of the network into several sub-networks, and then in each sub-network, each coordinator node adjusts the $\mathrm{BO}$ and $\mathrm{SO}$ values according to the energy level of the nodes in its sub-network. The authors of [5] propose a method: Coordinated Duty-cycle Algorithm (CDCA) which adapts the choice of the SO value according to the traffic and the priority of the packets (according to the nodes). More precisely, the algorithm compares the number of packets in the queue of a single node ( $n b P k t$ Wait) to the number of packets received $(n b P k t R)$ by the coordinator-node, if $n b P k t$ Wait is greater than nbpktR, it means that the traffic at that moment is very dense, and the current value of the active part (SO) of the super frame is not sufficient to satisfy the traffic level. Hence, it is necessary to increase the current value of SO.

In [3], Wang and Al. (2018) propose an algorithm for the adaptive performance management of WSN. This algorithm is based on the adaptation of the parameters macMinBE $(\mathrm{m} 0)$, macMaxCSMABackoffs (m) of the MAC layer. At the end of each Beacon Interval (BI), and according to: the number of nodes in its vicinity, the packet arrival, the delivery rate, and the transmission time, each node determines the adjustments of $\mathrm{m}$ and $\mathrm{m} 0$ to be made, to adapt to the current conditions of the network.

Furthermore, one of the problems that arise in LR-WPAN networks is the fact that even if the radio module of a node is in the inactive state, the collector module can still collect data that will be processed by the processing unit (uC) and stored in the buffer. When the radio unit will be active, these packets will then be processed for transmission. This packet storage generates a heavy load at the beginning of the active mode. This variation of the packet's number in the buffer affects the number of nodes that must compete to access the channel. It is then important to propose a model that deals with this fact for a better analysis. Zhuoling Xiao (2018) et Al [15] propose a performance evaluation of IEEE 802.15.4 in this sense. They model the state of the node based on the number of packets in its queue when it goes from inactive to active mode.

Although all these studies show the impact of the SO and BO parameters on the performance of the IEEE.802.15.4 network, they are still limited by their general nature because the proposed models do not explain and do not take into account the precise causes of the degradation as indicated by katayama (2019) in [16]. Indeed, uncertainties in the WSN are the main causes of the degradation of the network QoS, and they can be grouped into 3 types which are: uncertainties related to 1) the nodes, 2) the communication link and 3) the network traffic (network load). The authors of [16] are interested in the uncertainty related to radio links to improve network performance. For this purpose, they model the uncertainty of the links by a probabilistic model of fluctuation of the communication range of sensor nodes. Then they propose a packet aggregation technique to diminish the number of packets to be transported on the network. Their study is done using the default values of MAC parameters $(\mathrm{m} 0, \mathrm{~m}$, and $\mathrm{n})$ in a peer-to-peer topology, and all nodes are full function device (FFD) and non-beacon communication mode is used. However, it has been shown in [17] that the default values of $\mathrm{m}, \mathrm{m} 0$ and $\mathrm{n}$ cannot ensure an optimal QoS level. Furthermore, they do not consider the errors related to the PHY layer and also they neglect the probability of collisions. These limit this study because, in LR-WPAN, collisions cause huge losses in terms of power consumption, packet dropped, and delay. It is then necessary to take these into account for a better analysis of the network. Collisions are generally due to 2 situations:

- When at least 2 nodes randomly choose the same Backoff time value and start transmitting at the same time on the channel,

- Or when nodes are out of range and they cannot hear each other (listen), if they transmit at the same time, a collision will appear at the destination level.

To reduce collisions inside the network, Carrier Sense Multiple Access Collision Avoidance (CSMA/CA) has been defined. However, it is only effective for nodes that are not hidden. According to the study conducted in [18], the number of hidden nodes in the network can be around $41 \%$. However, the more the nodes are, more sooner collisions due to hidden nodes will be. Tseng et Al [19] propose a method to reduce the effect of hidden nodes in the network. It consists of grouping the nodes in clusters and subdividing the duration of the Super frame into several sub-periods according to the estimated number of hidden nodes. Although this method can reduce the impact of hidden nodes on the QoS, it remains limited because it only takes into account the new nodes integrating the network. It is possible that some already existing nodes in the network may not get along each other. Also in [20], Tallal et Al (2017) propose an analytical model for assessing the impact of hidden nodes on the reliability and lifetime of the IEEE 802.15.4 network. The model quantifies the hidden node problem in terms of the average percentage of hidden transceivers (nodes) relative to each sensor node in the network. According to this study, the higher the number of hidden nodes, the lower the probability of successfully transmitting a packet and the lower the lifetime of the network as indicated by Tseng et Al.[19].

QoS degradation is also due to the CSMA/CA access method. Indeed, the IEEE 802.15.4 MAC standard does not implement an effective mechanism to avoid collisions such as the RTS/CTS (Ready To Send / Clear To Send) mechanism in 802.11. Sending an RTS/CTS control frame before data transmission will be a huge burden since the size of the RTS/CTS frame is almost equal to the typical data frame size in WPANs [21], [22]. And several studies[20] [23], [24] have shown that if the channel state detection is not well managed, it leads to energy wastage due to a number of unnecessary CCAs and results in the degradation of the QoS. It should be noted that the IEEE.802.15.4 standard does not differentiate the channel occupancy by a data packet from a node and channel occupancy by an ACK packet from the receiver. Once the channel is declared as busy, this induces the nodes to go into sleep mode for a given period which could be even longer because the Backoff Exponent (BE) number is incremented. 


\section{Wireless Sensor Node Performance Analysis Under Mac Protocol IEEE 802.15.4}

This has the effect of increasing the losses rate, the delay and the reliability in the network. To address this problem, Son et Al (2016) [6] propose a method based on the principle of listening segmentization (CCA) of the channel to detect whether the channel is busy by an Ack or data (Fig. 1-B). Also in order to detect the channel status, Yundra et Al (2018) propose in [25] a modification of the IEEE 802.154 channel access mechanism. In this study, the number of CCAs is set to 3 instead of 1 or 2 as observed in most of the studies [2] [23]. However, the greater the number of CCAs, the greater the energy consumption relative to the CCAs, which limits this study. Sahoo et Al (2019) [24] also propose a new channel access mechanism. The novelty in this protocol is the introduction of signaling messages at the start/end of transmission as well as before the emission of an ack. This approach is intended to prevent nodes from performing unnecessary listening (CCAs) that would result in energy consumption. These signals allow the other nodes which have detected them to go into idle mode for a time that they will have determined according to the signal. This allows them to know when they will have to wake up to perform their CCA, without increasing their window size (BE and NB).

However, it should be noted that once the node has waited (inactive) for the time determined thanks to the detected signal message, it is likely that another node which did not receive the signal will become active and access to the channel before the expiration of the waiting time of the node which having received the signal. When this node wakes up, it will then find that the channel is occupied and it will perform a new Backoff mechanism. This increases the delay and the packet losses rate as the waiting time for a packet in the buffer will be longer. This leads to high power consumption and then reduces the network's lifetime.

The authors of [26] have conducted a study on the performance of the LR-WPAN network, based on the Park [7] and Zayani [8] studies. In [8], the authors have modified the model proposed by Park [7] for a better analysis of the 802.15.4 standard. They integrate the probability of failure due to errors in the channel and transmission mechanisms at the PHY layer (Encoding, modulation, etc.). More precisely, they include the probability that a node will have data to transmit because only nodes with data will compete for channel access. This was not taken into account by Park, who considered that all nodes competed to access the channel, but this is not the exact. Based on these 2 studies, Kone et Al [26] propose, in addition to reliability, delay, and throughput, to study an additional metric, which is the energy consumed by the sensor in all the modules. In fact, in addition to the energy dissipated in the transmission module, he adds the energy dissipated in the data collector and processing modules to have a better knowledge of the node behaviour. Although this study makes it possible to analyze and know the energy consumption of a given node in the network according to the MAC layer parameters $(\mathrm{m}, \mathrm{m} 0, \mathrm{n})$, it remains limited because it does not differentiate the channel occupancy during the second CCA by an ack message and a data, and it does not consider the hidden nodes inside the network.

This paper aim to analyze the performance of the IEEE 802.15.4 MAC standard. We propose to extend the work carried out by Park, Zayani and Kone through an analysis of the performance of the IEEE 802.15.4 MAC standard that takes into account the above problems.

The main contribution of this paper is to propose an analytical model of the MAC protocol 802.15.4 standard which considers the hidden nodes, and explains the channel occupancy by an acknowledgement message (ack) or data to the 1st and 2nd CCA.

\section{PROPOSED MODEL}

In this section, we present our analytical model. It is a generalized model of the CSMA/CA mechanism of the IEEE 802.15.4 standard, with a limited number of packet retransmission. Our objective is to provide a tool for evaluating this wireless communication standard to allow its appropriate use in different application domains.

Our approach is based on the work of [7], [8], [26]. The developed model that we propose, analyses a PHY and MAC IEEE 802.15.4 layer channel in which several (N) unsaturated stations compete to communicate with a coordinator node. In [7], Park et al. then Zayani et Al in [8], model the exponential backoff process by a Markov chain taking into account the number of retransmission limits, and the unsaturated traffic. Park [7] and Zayani [8] define the probabilities for the following events: a node attempts a first listening of the carrier to determine the state of the channel ( $\tau$ ), a node detects that the channel is busy during the first CCA1 ( $\alpha$ ) or a node detects that the channel is busy during the second CCA2 ( $\beta$ ). Based on these probabilities, some simple and efficient approximation of reliability, delay and power consumption under low traffic conditions are proposed. These works inspire the author of [26] who have proposed a detailed analytical model of energy consumption of a sensor node, by adding to the energy consumption of the transceiver module, those related to the collecting and processing modules. This model gives the energy consumption as a function of the MAC layer parameters (m, $\mathrm{m} 0, \mathrm{n}$ ) and the application layer parameters which are represented here by the load per node and the number of nodes in the network.

As we are concerned, we think that this is a good basic model to know the behaviour of each node in the IEEE 802.15.4 network and to adapt the QoS level according to these parameters. However, it should be noted that their model remains limited in terms of precision, because in their studies, the authors of [7], [8], [26] do not consider the hidden nodes, which have a real influence on the network QoS as we mentioned in the previous section. Moreover, their models certainly bring out the probabilities encountered at the first CCA1 $\left(\alpha_{1}, \alpha_{2}\right)$ but they do not detail those related to the second CCA2 ( $\beta_{1}$ and $\beta_{2}$ ), which are respectively the probabilities that the channel is occupied by a data and an acknowledgment message (ack) during the second CCA2. In [27], a model that highlights the impact of hidden nodes is proposed by Pattanaik et Al. However, the authors did not consider the impact of the limit number of retransmissions and also they did not deals with the remarks made by Zayani et al in [8].

So to have a more precise and accurate model, we propose in our approach to consider these above elements that we have indicated. 
Let denoted by $\mathrm{N}$ the total number of nodes in the network, and ph the percentage of hidden nodes. Then for a given node, the number of hidden nodes is N.ph and the number of nodes in its line of sight is (1-ph)N. It is important to know that in our proposed model and contrary to Park's model, the detection of the channel occupation by an ACK ( $\alpha_{2}$ and $\left.\beta_{2}\right)$ allows a node to not perform another unnecessary backoff. This avoids energy losses as indicated by the authors of [6].We are inspired by the Markovian model proposed in [7] to make some modifications. Furthermore, we introduce into equations (1) and (2) and (3) deduced from the Markovian model proposed by Park [7] and taken up by Zayani [8] and Kone [26], the number of nodes N(1-ph) visible by a node and the number of hidden nodes (N.ph) and we give the expressions of $\tau, \alpha_{1}, \alpha_{2}, \beta_{1}$ and $\beta_{2}$. The new model is shown in the following Fig. 2.

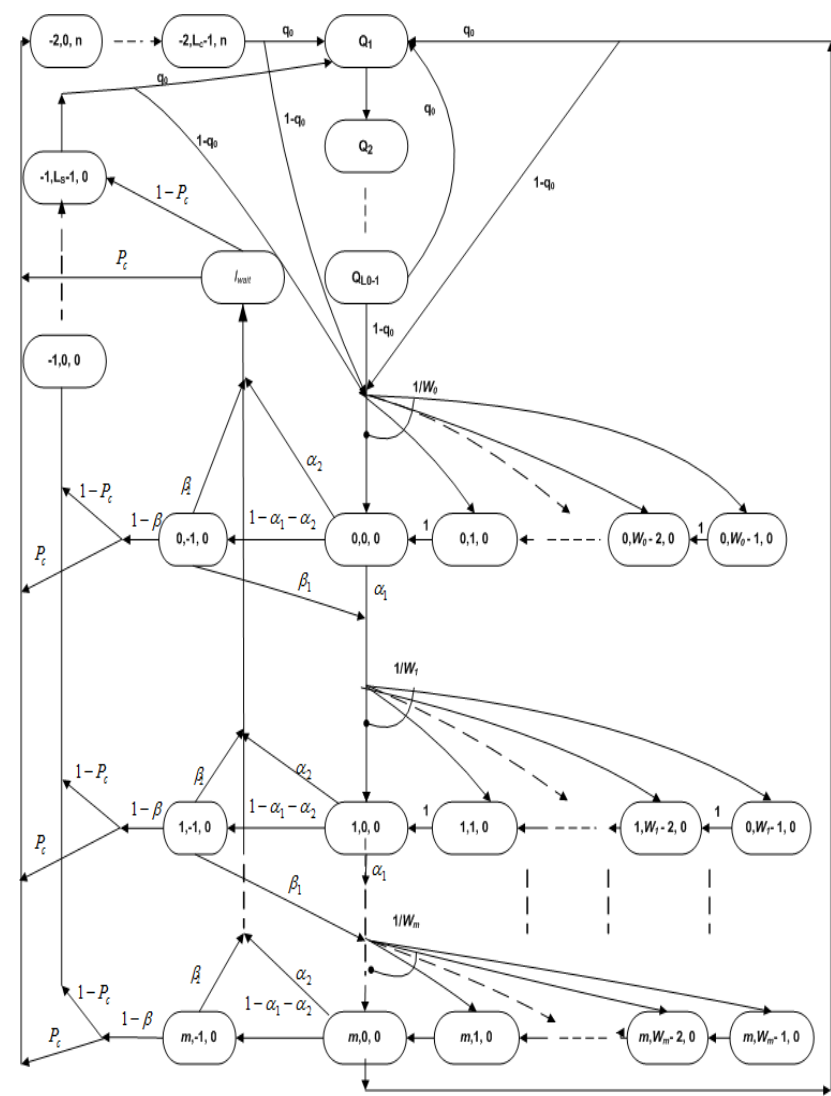

Fig. 2.Proposed Markov chain model for CSMA/CA algorithm for IEEE 802.15.4

In this model, $b_{i, k, j}$ is the probability of the state of the system associated with the Markov chain $\mathrm{M} / \mathrm{M} / 1 / \mathrm{K}$ describing the IEEE 802.15.4 beacon standard. $i, j$, and $k$ are successively the variables that denote the backoff stage, the state of the backoff counter and the state of retransmission counter, respectively. With i belongs to $[-2 ; m]$, $\mathrm{k}$ belongs to $[-1$; $\max (W i-1, L s-1, L c-1)$ and $\mathrm{j}$ belongs to $[0 ; \mathrm{n}] . I_{\text {wait }}$ is the waiting state for a given node when it detect that the channel is occupied by an ACK message.

The analytical expressions below are different from those in [27] by the fact that we have replaced $\tau$ with $\left(1-p_{0}\right) \tau$. Because only the nodes with data, will compete to access the channel, and also we have considered the number of retransmissions $(n)$ as in [7].
We have:

The busy channel probability during the first CCA:

$\alpha=\alpha_{1}+\alpha_{2}$

With

$\alpha_{1}=L_{D}\left[1-\left(1-\left(1-p_{0}\right) \tau\right)^{N(1-p h)-1}\right]\left[\alpha_{2}+\left(1-\alpha_{1}-\alpha_{2}\right)\left(1-\beta_{1}\right)\right]$ )

$$
\begin{aligned}
& \alpha_{2}=L_{a c k} \cdot \frac{N\left(1-p_{0}\right) \tau\left(1-\left(1-p_{0}\right) \tau\right)^{N(1-p h)-1}}{1-\left(1-\left(1-p_{0}\right) \tau\right)^{N(1-p h)}} \\
& \cdot\left(1-\left(1-\left(1-p_{0}\right) \tau\right)^{N(1-p h)-1}\right)\left(1-\alpha_{1}-\alpha_{2}\right)\left(1-\beta_{1}-\beta_{2}\right)
\end{aligned}
$$

The busy channel probability during the second CCA:

$\beta=\beta_{1}+\beta_{2}$

With

$$
\begin{aligned}
& \beta_{1}=\frac{1-\left(1-\left(1-p_{0}\right) \tau\right)^{N(1-p h)-1}}{2-\left(1-\left(1-p_{0}\right) \tau\right)^{N(1-p h)}} \\
& \beta_{2}=(N(1-p h)-1)\left(1-p_{0}\right) \tau\left(1-\left(1-p_{0}\right) \tau\right)^{N(1-p h)-2} \\
& \cdot\left(1-p_{e}\right)\left(1-\left(1-p_{0}\right) \tau\right)^{2 L_{D} N p h} \cdot\left(\alpha_{2}+\left(1-\alpha_{1}-\alpha_{2}\right)\left(1-\beta_{1}\right)\right)
\end{aligned}
$$

With $\alpha$ and $\beta$, we then deduce the busy channel probability $x:$

$x=\alpha+(1-\alpha) \beta$

The probability that a node attempts is first CCA:

$$
\begin{aligned}
& \tau=\left(\frac{1-x^{m+1}}{1-x}\right)\left(\frac{1-y^{n+1}}{1-y}\right) b_{0,0,0} \\
& y=P_{\text {fail }}\left(1-x^{m+1}\right)
\end{aligned}
$$

$L_{D}$ and $L_{A c k}$ are respectively the data packet length and acknowledgment length in number of slots, $P_{0}$ is the stationary probability of having an empty queue, i.e. the probability that the node has no packets to transmit (an estimation is given in [8]). $m$ is the maximum number of backoff stage that a node can perform before declaring that the channel access attempt has failed; $n$ is the maximum number of attempts allowed after a transmission failure; $P_{e}$ is the probability relating to transmission errors due to the transmission channel constraints and the modulation/coding technics used. $b_{0,0,0}$ is the state where $i=j=k=0$. An approximation of this value is given in [7].

The transmission failure probability is $P_{\text {fail }}$ :

$P_{\text {fail }}=1-\left(1-P_{k}\right)\left(1-P_{\text {col }}\right)\left(1-P_{e}\right)$

With $P_{c o l}$ the probability that a collision occurred.

$P_{\text {col }}=1-\left(1-\left(1-p_{0}\right) \tau\right)^{N-1}$ 


\section{Wireless Sensor Node Performance Analysis Under Mac Protocol IEEE 802.15.4}

We deduce the reliability $(R)$ :

$R=1-P_{c r}-P_{c f}$

Where $P_{c r}$ and $P_{c f}$ are respectively the probability that packet is discarded due to retry limits and the channel access failure probability.

$$
\begin{aligned}
P_{c r} & =y^{n+1} \\
P_{c f} & =\frac{x^{m+1}\left(1-y^{n+1}\right)}{1-y}
\end{aligned}
$$

We deduce also the delay (D):

$D=\frac{L}{\lambda\left(1-P_{k}\right)}$,

Where $P_{k}$ is defined as the probability of having full buffer and $\lambda$ (frames/s) is the packet arriving rate in each the buffer queue, and it follows a Poisson arrival process. Note that the energy consumption model we use for our study here is based on the proposed equations (2) in [26]. More details could be find in [26].We integrate in these models our expressions of: $\tau, \alpha_{1}, \alpha_{2}, \beta_{1}$ and $\beta_{2}$. The new model of energy consumption at the radio module is:

$$
E_{R F_{-} \text {unit }}=E_{b}+E_{s c}+E_{t}+E_{q}+E_{h}+E_{w}
$$

Where:

$E_{b}$ is the average energy consumption of RF during idle backoff state

$$
\begin{aligned}
& E_{b}=P_{i} S_{b} \sum_{i=0}^{m} \sum_{k=1}^{W_{i}-1} \sum_{j=0}^{n} b_{i, k, j}= \\
& \frac{P_{i} \cdot \tau}{2}\left[\frac{(1-x)\left(1-(2 x)^{m+1}\right)}{(1-2 x)\left(1-x^{m+1}\right)} \cdot 2^{m_{0}}-1\right] \cdot S_{b}
\end{aligned}
$$

$E_{s c}$ is the average energy consumption of RF during channel sensing state

$$
\begin{aligned}
& E_{s c}=P_{s c} S_{b} \sum_{i=0}^{m} \sum_{j=0}^{n}\left(b_{i, 0, j}+P_{i} \cdot b_{i,-1, j}\right)= \\
& P_{s c} \cdot \tau \cdot\left(2-\left(\alpha_{1}+\alpha_{2}\right)\right) \cdot S_{b}
\end{aligned}
$$

$E_{t}$ is the average energy consumption of RF during packet transmission stage

$$
\begin{aligned}
& E_{t}=P_{t} \cdot S_{b} \cdot \sum_{i=-2}^{-1} \sum_{k=0}^{L-1} \sum_{j=0}^{n} b_{i, k, j}+P_{i} \cdot S_{b} \cdot \sum_{i=-2}^{-1} \sum_{j=0}^{n} b_{i, L, j}+ \\
& S_{b} \cdot \sum_{i=0}^{n} \sum_{k=L+1}^{L+L_{\text {ack }}+1}\left(P_{r} \cdot b_{-1, k, j}+P_{r} \cdot b_{-2, k, j}\right)= \\
& \left(1-\left(\alpha_{1}+\alpha_{2}\right)\right)\left(1-\left(\beta_{1}+\beta_{2}\right)\right) \cdot \tau \cdot \\
& {\left[P_{t} \cdot L+P_{i}+\left(P_{r} \cdot\left(1-P_{\text {fail }}\right)+P_{i} \cdot P_{\text {fail }}\right) \cdot L_{a c k}\right] \cdot S_{b}}
\end{aligned}
$$

$E_{q}$ is the average energy consumption of RF during idle stage without generation packet

$$
\begin{aligned}
& E_{q}=P_{s p} \cdot S_{b} \sum_{l=0}^{L_{0}-1} Q_{l}=P_{s p} \cdot \frac{1-p_{0}}{p_{0}} \cdot L_{0} \cdot b_{0,0,0} \cdot S_{b} . \\
& {\left[x^{m+1} \cdot(1+y)+\left(P_{\text {fail }} \cdot y^{n}+\left(1-P_{\text {fail }}\right) \cdot(1+y)\right) \cdot\left(1-x^{2}\right)\right]}
\end{aligned}
$$

$E_{h}$ is the average energy consumption of RF during packet copying stage

$$
\begin{aligned}
& E_{h}=P_{s p} \cdot S_{b} \sum_{l=0}^{L_{1}-1} H_{l}=P_{s p} \cdot L_{1} \cdot b_{0,0,0} \cdot S_{b} . \\
& {\left[x^{m+1} \cdot(1+y)+\left(P_{\text {fail }} \cdot y^{n}+\left(1-P_{\text {fail }}\right) \cdot(1+y)\right) \cdot\left(1-x^{2}\right)\right]}
\end{aligned}
$$

$E_{w}$ is the average energy consumption of RF during wake-up state after being idle during backoff period

$E_{w}=P_{w} \cdot S_{b} \cdot\left(1-p_{0}\right) \cdot Q_{L_{0}-1}=$

$P_{w} \cdot p_{0} \cdot b_{0,0,0} \cdot S_{b} \cdot\left[\begin{array}{l}x^{m+1} \cdot(1+y)+\left(P_{\text {fail }} \cdot y^{n}+\left(1-P_{\text {fail }}\right) \cdot(1+y)\right) \cdot \\ \left(1-x^{2}\right)\end{array}\right]$

$P_{i}, P_{s c}, P_{t}, P_{r}, P_{w}$, and $P_{s p}$ are respectively: the power consumption of RF in idle-listen state, channel sensing state, transmitting state, receiving state, wake-up state and sleep states during the backoff stages.

\section{SIMULATION AND RESULTS}

In this section, we present and analyze the results of the simulation of our proposed model. For a better analysis of results, and to show the accuracy (efficiency) of our contribution, we use the same experimental conditions as in [9].The simulation parameters are presented in tables 1 and 2 .

Table-I: Main parameters used in PHY layer [9].

\begin{tabular}{|c|c|}
\hline Paramters & Values \\
\hline Noise Figure & $13 \mathrm{~dB}$ \\
\hline Pathloss exp & 2 \\
\hline Noise & $15 \mathrm{~dB}$ \\
\hline Shadowing std. dev & $23 \mathrm{~dB}$ \\
\hline Bandwidth & $30 \mathrm{Khz}$ \\
\hline STD Tx power & 10 \\
\hline Data Rate & $19.2 \mathrm{kbit} / \mathrm{s}$ \\
\hline Max Tx range & $150 \mathrm{~m}$ \\
\hline
\end{tabular}

Table-II: Power consumption of different operation modes of Waspmote [9]

\begin{tabular}{|c|c|}
\hline Operation mode & $\begin{array}{c}\text { Power consumption } \\
(\mathrm{mW})\end{array}$ \\
\hline$P_{r}$ & 166.518 \\
\hline$P_{t}$ & 346.5 \\
\hline$P_{w}$ & 150.348 \\
\hline$P_{i}$ & 2.343 \\
\hline$P_{s p}$ & 0 \\
\hline
\end{tabular}

The numerical performance results presented here were obtained through a Monte Carlo simulation of 3 sizes of star networks (10, 50 and 100 nodes). The performance criteria we use to evaluate the proposed model are reliability (the probability that a packet transmitted by a node arrives at the coordinator node without collision or transmission errors), delay (the average time between the transmission and reception of a packet) and power consumption of the radio module of a given node. 
These analyses are based on the MAC parameters $(m, m 0, n)$ and the density of node. For a better understanding of the influence of these MAC parameters, we choose to go beyond the predefined limit values of the IEEE 802.15.4 standard. In all figures below, the left side of the pink vertical line represents the results related to the default values of the MAC parameters specification in the IEEE 802.15.4 standard.

We note that in general, the QoS of the network degrades with the density of the nodes.

\section{A. Reliability}

Fig. 4 displays the evolution of reliability as a function of the MAC parameters $\left(m_{0}, m, n\right)$ and the density of nodes. In general, we can see that reliability degrades when the number of nodes attached to the coordinator node increase. Indeed, the greater the number of nodes, the more nodes will try to access the channel to transmit their packet. This leads to a higher channel access failure rate resulting in packet losses. Also as indicated [19], the greater the number of nodes inside the network, the higher the packet losses due to collisions will be, as the probability of nodes transmitting at the same time, as well as the probability of nodes which not hear each other, become higher. This explain that degradation with density.

In Fig. 4A, we observe that as the value of $m_{0}$ increases, reliability increases and tends towards 1 regardless of the size of the network. This evolution can be explained by the fact that a high value of $m_{0}$ reduces not only the probability of packet collisions due to simultaneous access to the channel by at least 2 nodes wishing transmit but also reduces the packet dropped rate due to the limitation of attempts allowed to access the communication channel. The larger the $m_{0}$, the larger the size of the contention window $\left[0 ; 2^{\wedge \mathrm{m}_{0}}-1\right]$ will be. This reduces the probability that two nodes will choose the same value in this interval, thus increasing the rate of channel access by a given node.

Also, we observe in Fig.s $4 B$ and $4 C$, that as the values of $m$ and $n$ increase, reliability also increases up to a certain level and then decreases rapidly for the dense network $(\mathrm{N}=100$

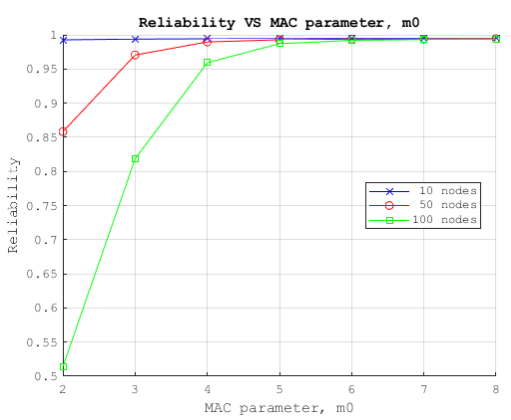

A

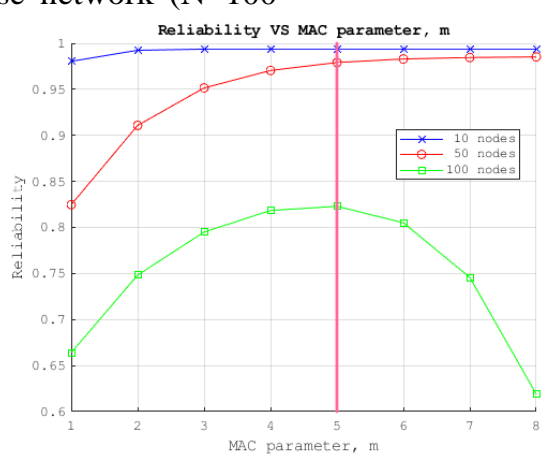

B nodes). This decrease is due not only to a high probability of losses caused by channel access failure, but also to a high rate of packet dropped due to retransmission limit. Indeed, inside a dense network, retransmission is more important because of the frequent occurrence of collision due to the transmission of hidden nodes (Fig. 11). So the higher the value of $m$, the greater the number of nodes that find the channel busy and also the higher the probability of packets being dropped due to channel access failure as it can be seen in Fig. 5 below. However, we can notice that in our model this decrease is less accentuated than the model of Zayani et Al [8]. Indeed, this situation can be explained by the fact that their model does not distinguish the channel occupation by an ACK and a data. This leads nodes to perform more regular backoff operations even if it is an ACK, which is circulating on the channel. This increases then, the number of backoff which lead to a higher packet losses due to channel access failure as can be seen in Fig. 6B.

Also for higher values of $n$, there will be a higher number of retransmissions, which reduces the losses due to reaching the maximum number of retransmissions, resulting in a slight increase of the reliability. However, it should be noted that retransmissions are due to packet collisions or errors. The denser the network size, the greater the probability of collision, thus reducing reliability in the case of dense networks. Therefore, having a high number of retransmissions ( $n$ ) and channel access attempts is not sufficient to ensure high reliability in large networks. Our analysis confirm that of Kone in [26].

In conclusion, we can say that for a dense network, only $m_{0}$ has a great influence on the reliability. But when the size of network is not dense ( $N \prec 100$ ) one could also deal with the parameter $m$ to have a high level of reliability.
A

(1)

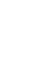




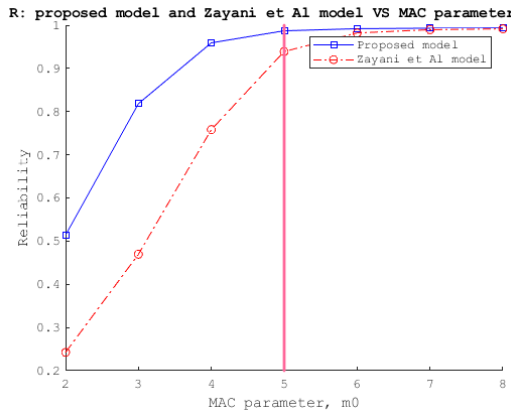

D

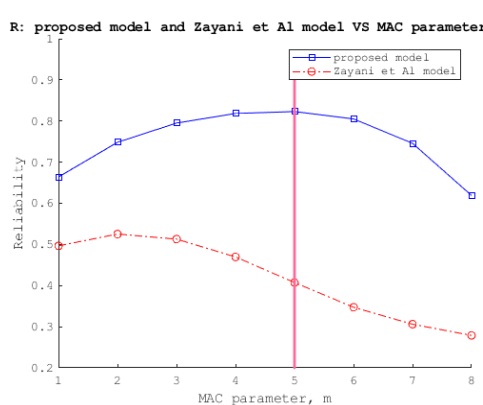

E

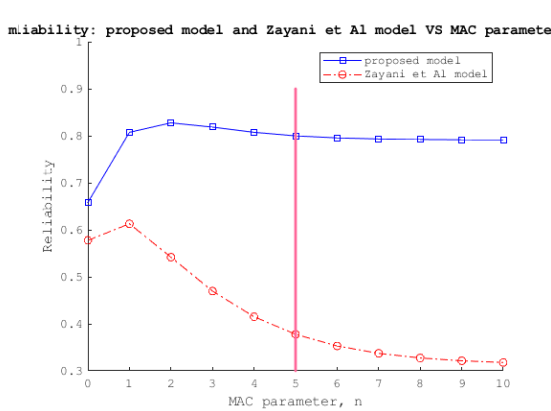

F

Fig. 3.Reliability as a function of MAC parameters $: m 0=1 \ldots 8, m b=8, m=0 \ldots 10, n=0 \ldots 10, N=10,50,100:(A$ and D) $\mathrm{m0}=2 . .8, \mathrm{mb}=8, \mathrm{~m}=4, \mathrm{n}=3 ;(\mathrm{B}$ and $\mathrm{E}) \mathrm{m}=1 \ldots 8, \mathrm{m0}=3, \mathrm{mb}=8, \mathrm{n}=3$ and $(\mathrm{C}$ and $\mathrm{F}) \mathrm{n}=0 . .10, \mathrm{m0}=3, \mathrm{mb}$ $=8, \mathrm{~m}=4$. In $\mathrm{D}, \mathrm{E}$ and $\mathrm{F}, \mathrm{N}=100$.

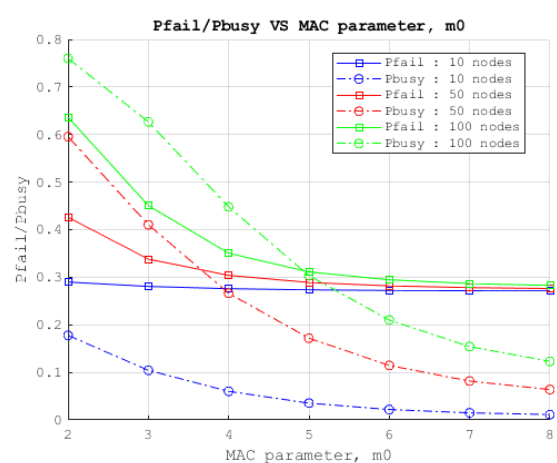

A

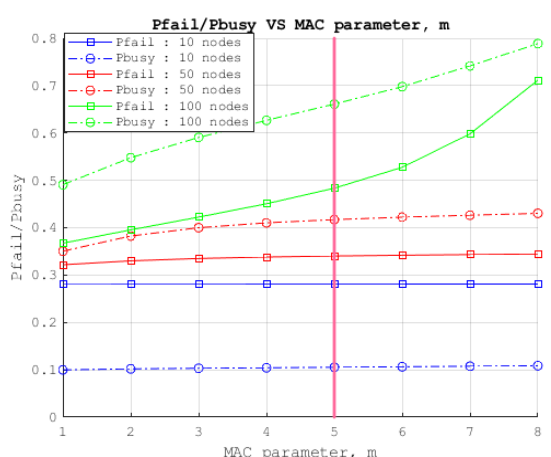

B

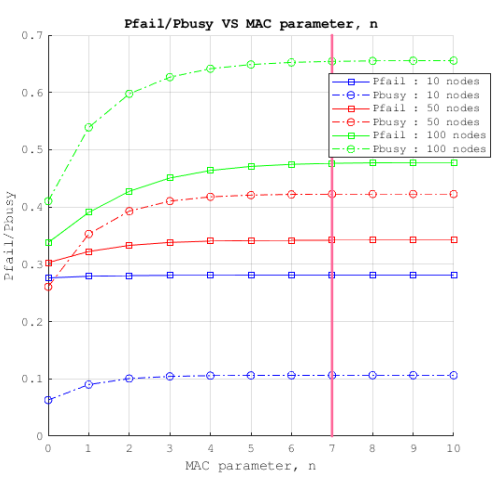

C

Fig. 4. Transmission failure probability and busy channel probability vs $\mathrm{MAC}$ parameters: $\mathrm{m}=1 \ldots 8, \mathrm{m0}=3, \mathrm{mb}=8, \mathrm{n}$ $=3 . N=10,50$, and 100 .

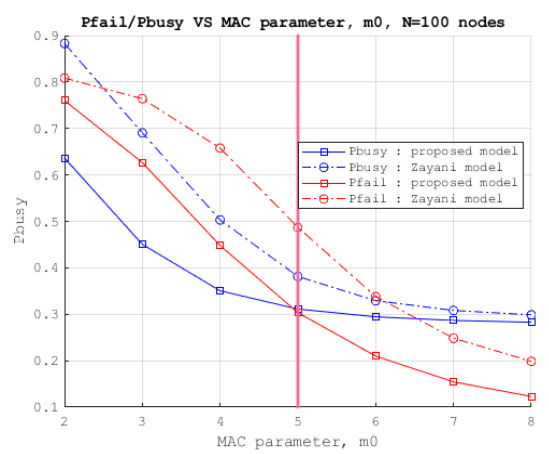

A

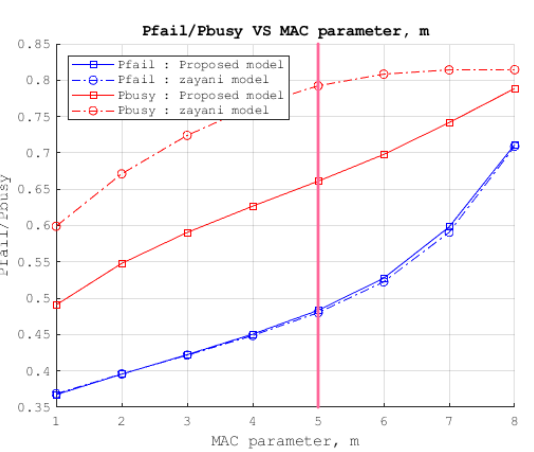

B

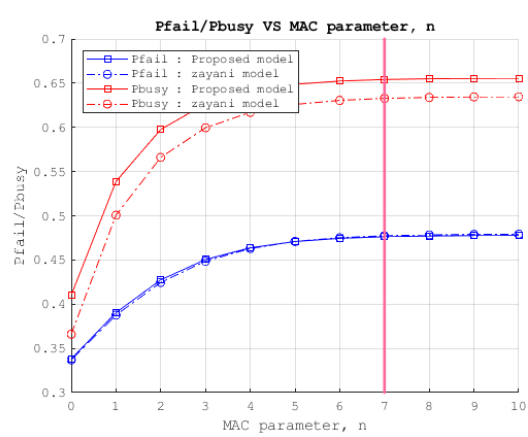

C

Fig. 5.Comparison of transmission failure probability and busy channel probability vs. MAC parameters : $\mathrm{m0}=1$ to 8 , $m b=8, m=0 . .10, n=0 . .10, N=100:(A) m 0=2 \ldots 8, m b=8, m=4, n=3 ;(B) m=1 . .8, m 0=3, m b=8, n=3 ;(C)$ $\mathrm{n}=0 . . .10, \mathrm{m0}=3, \mathrm{mb}=8, \mathrm{~m}=4$. 


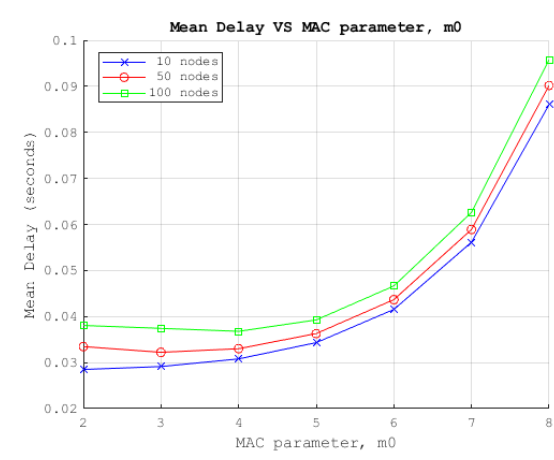

A

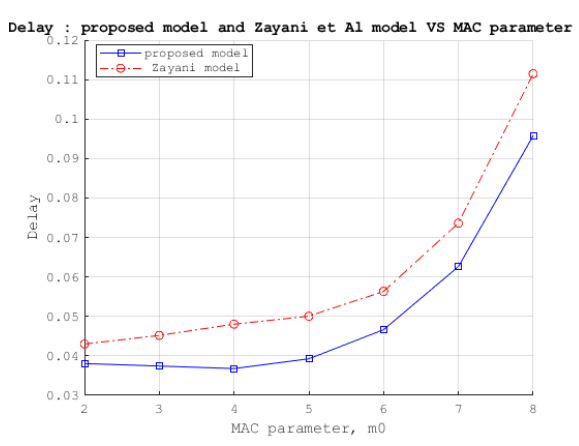

$\mathrm{D}$

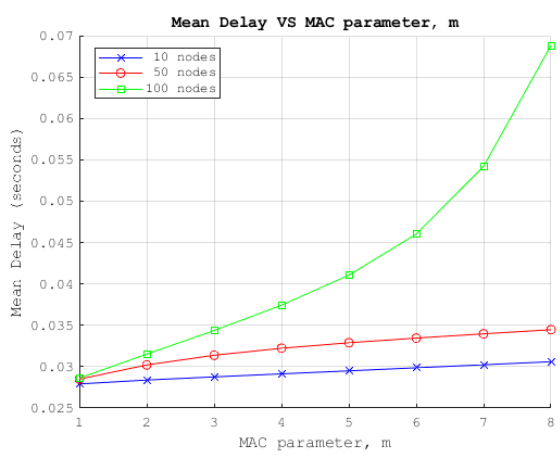

B

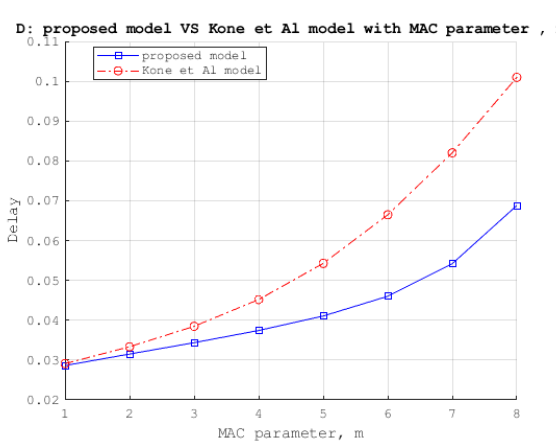

$\mathrm{E}$

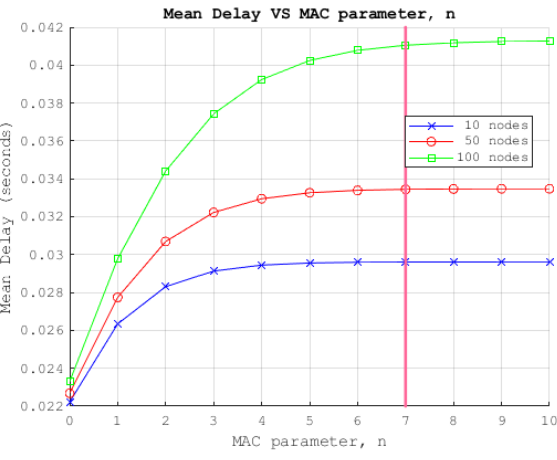

C

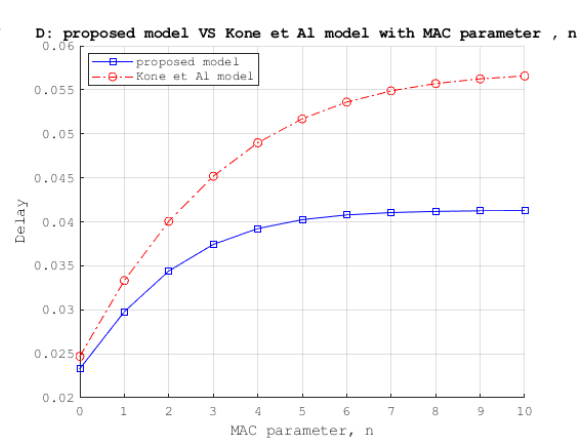

F

Fig. 6. Evolution of delay as a function of MAC parameters $: m 0=1 \ldots 8, m b=8, m=0 \ldots 10, n=0 \ldots 10, N=10,50$ and 100: $(A$ and $D) m 0=2 . .8, m b=8, m=4, n=3 ;(B$ and $E) m=1 \ldots 8, m 0=3, m b=8, n=3$ and $(C$ and $F) n=0 \ldots 10$, $\mathrm{mo}=3, \mathrm{mb}=8, \mathrm{~m}=4$. for $\mathrm{D}, \mathrm{E}$ and $\mathrm{F}, \mathrm{N}=100$.

\section{B. Delay}

Fig.7 shows the evolution of the average delay for transmitting a packet as a function of network density. In general, we observe that the larger the size of the network, the greater the delay is.

More particularly, we can notice in Fig. 7A, an exponential increase of delay with $m_{0}$ whatever the size of the network. This exponential evolution is explained by the fact that the backoff time is calculated using the binary exponential backoff $(B E B)$ algorithm. In addition, the more $m_{0}$ increases, the more the time spend by a given node in idle state will increase since the window size will increase exponentially when the node finds the channel busy.

In Fig. $7 B$, we note that for dense networks $(\mathrm{N}=100)$, the average delay increases exponentially with the values of $m$, while for the networks where the number of node is less than 100 , the variation is not significant. Indeed, the increase in $m$, means that there is more nodes who go to the idle mode due to the channel access failure and collisions which are more frequent in dense networks especially with the presence of hidden nodes. This leads to longer idle-backoff times, resulting in a higher delay. So we can say that $m$ has an influence on the delay only when the number of node is higher than 50, unlike Kone[26] who considers that for the value of $m=50$ the impact of $m$ was relevant.

Also, we note in Fig. $7 \mathrm{C}$ that the smaller the size of the network, the shorter the delay, and it grows weakly for high values of $n$ until it reaches a saturation point. This can be explained by the fact that in small networks, (for example, $N$ $=10$ nodes), a higher value of $n$ has no significant impact on the number of packet retransmissions. Because the probability that a node finds the busy channel under these conditions is low (Fig. 5C and 6c) and the collision rate is also weak Fig. 11. However, retransmissions are due to collisions and packet transmission errors. Hence, a short variation of the average packet transmission delay in small networks for high values of $n$. For large networks ( $N \geq 50$ ), we also see that the delay increases slightly to a saturation point from which it remains constant. More the size of network is, greater will be the delay. This is due to the hidden node as we have explained in the preview section.

In addition, when we compare our model to that of Zayani et al [8], we notice in general that, this growth of the delay according to the size of the network is less accentuated with our model contrary to that of Zayani as indicated in Fig. 7D, $7 E$ and $7 F$. This is explained by the fact that, unlike Zayani[8], our model distinguishes the occupation of the channel by an Ack and data. This distinction allows the node to avoid performing a backoff procedure, which generates an additional waiting time when the channel is occupied by an Ack. We conclude that in large networks $(N=100), m_{0}$ and $m$ are the key parameters with respect to $n$. But when the size of network is less than 100 nodes, only $m_{0}$ has a relevant impact on the delay. This analysis extend those of Kone and Zayani who considers that $m_{0}$ is the key parameter only when the number of nodes is under 50 . 


\section{Wireless Sensor Node Performance Analysis Under Mac Protocol IEEE 802.15.4}

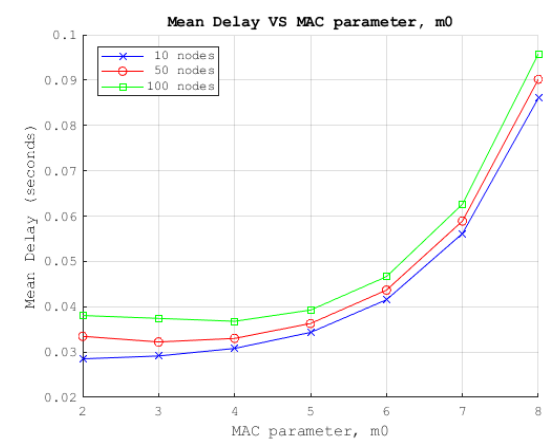

A

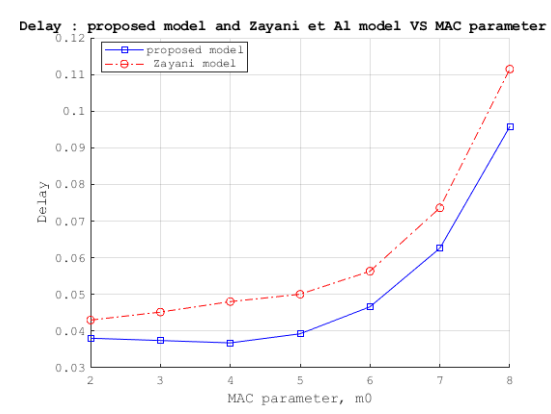

D

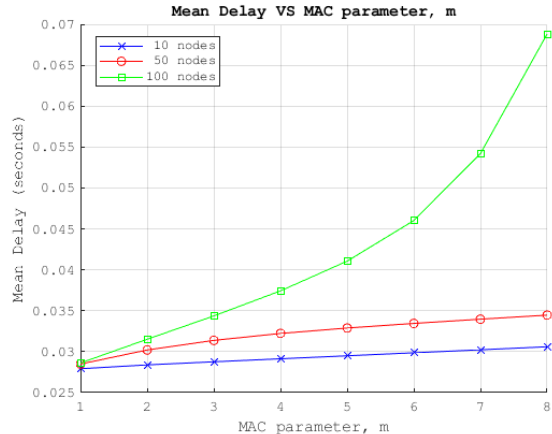

B

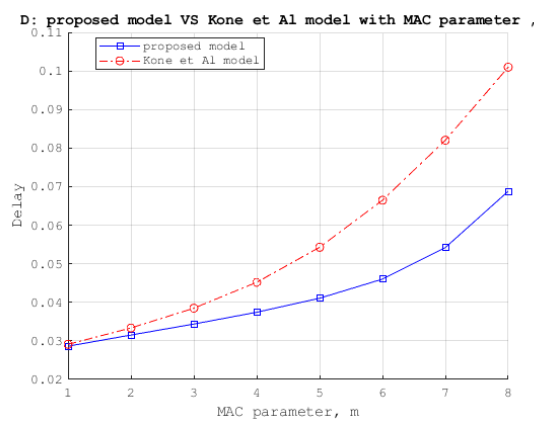

$\mathrm{E}$

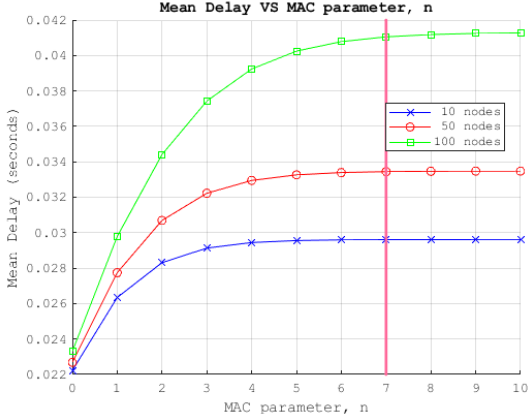

C

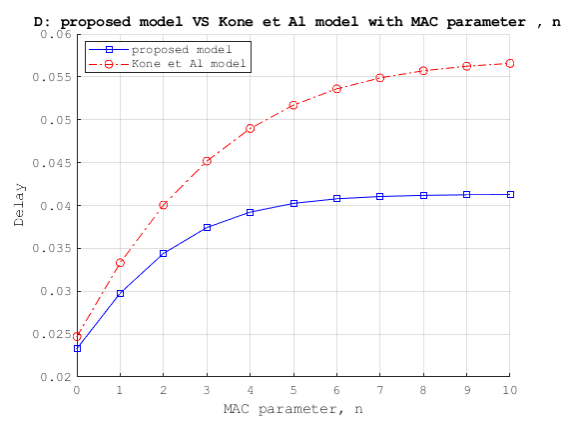

$\mathrm{F}$

Fig. 7.Evolution of delay as a function of MAC parameters $: m 0=1 \ldots 8, m b=8, m=0 \ldots 10, n=0 \ldots 10, N=10,50$ and 100: $(A$ and $D) m 0=2 \ldots 8, m b=8, m=4, n=3 ;(B$ and $E) m=1 \ldots 8, m 0=3, m b=8, n=3$ and $(C$ and $F) n=0 \ldots 10$, $\mathrm{mo}=3, \mathrm{~m}_{\mathrm{b}}=8, \mathrm{~m}=4$. for $\mathrm{D}, \mathrm{E}$ and $\mathrm{F}, \mathrm{N}=100$.

\section{Energy Consumption}

In Fig. 8, we have the average energy dissipated by the radio module of a node (equation 17 ). Here, each operation related to an attempt transmission takes place in a time interval called backoff period $\left(S_{b}\right)$ chosen randomly as indicated by the IEEE 802.154 standard. In Fig. 8 we could note that in general, the average energy consumption decreases as the number of nodes $N$ increases.

Indeed, the higher the value of node, the greater the probability that the channel will be busy as well as the waiting time of a node in the idle backoff stage and therefore, the activations of the radio transmitter will be smaller. More specifically, in Fig. 8A we observe that the more the value of $m_{0}$ increases, the more the energy consumed decreases exponentially. This decrease is explained by the fact that the higher the $m_{0}$, the higher the average time that a node will spend in idle backoff stage; this means that the radio module will consume less energy, that's why we have this reduction in the energy consumption.

In addition, we notice in Fig. $8 B$ that when the size of the network is small ( $N \leq 50$ ) and if $m$ increases, the average energy consumption of a node by slot varies slightly and it is about 9 and 5 micro joules respectively for $N=10$ and $N=50$. However, we see that the larger the size of the network ( $N=100$ ), the more the energy consumption is influenced by the values of $m$ (Fig. $8 E$ ).

Indeed, as well with the model proposed by Zayani et al[8], as ours, we can see that for a larger network ( $N=100$ ), when $m$ increases, the average energy consumption decreases slowly respectively for $m \leq 4$ (Zayani et al[8]) and $m \leq 5$ (proposed model) then increases for $m \succ 4$ and $m \succ 5$ (values outside the range defined by IEEE 802.15.4 specifications). More particularly we can see that a node in a network of 100 nodes, consumes less energy than a node in a network of 50 for $m \leq 5$. This is explained by the fact that more the number of nodes is small, the higher the channels access probability will be, so that the nodes in the networks of small sizes will tend to be more active for the sending of the data while the nodes in the dense networks will most often find that the channel is busy (Fig. 5B), so these will go more to the idle stages, which allows them to reduce their energy consumption. Also in Fig. 8D, we can see that energy consumption is more significant with our model when $m \leq 5$. This situation is explained by the fact of hidden nodes inside the network (here $P_{h}=0.41$ as mentioned in [19]). Indeed, the higher the number of hidden nodes, the greater the energy consumption as we can see in Fig. 9. This is because of collision due to the transmission of the hidden node which occurred more.

However, it should be noted that for $m \succ 5$, the average energy consumption in the case of our model is lower than that of the model proposed by Kone et Al[26]. This is because the probability that the channel is occupied in ours is less than that of Park [7] used by Kone as it can be seen in the Fig. 10. In fact, the lower the probability that the channel is busy, the lower the carrier sensing process by a given node. Then the energy consumption induced by this process of determining the state of the channel will also be lower.

Fig. 8C shows us the evolution of energy consumption as a function of parameter $n$. We notice in general that consumption varies very weakly with values of $n$. 
However, we observe a slight decrease in energy consumption up to a certain level (which depends on the size of the network) before remaining constant regardless of the value of $n$. For example, in large networks $N=100$, this decrease is because, the probability that a node finds the channel busy is high in this phase as shown in Fig. 5C. This leads the nodes to be most often in idle mode, which thus reduces their energy consumption.

To conclude we can say that $m_{0}$ and $m$ have more impact on the average energy consumption than $n$. So one could deals with both to a better management of energy consumption of a node in LR-WPAN.

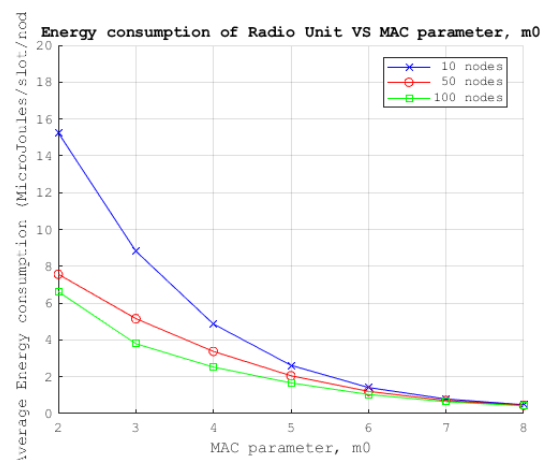

A

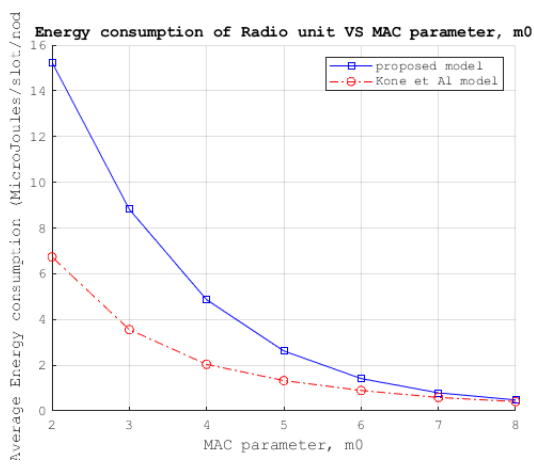

$\mathrm{D}$

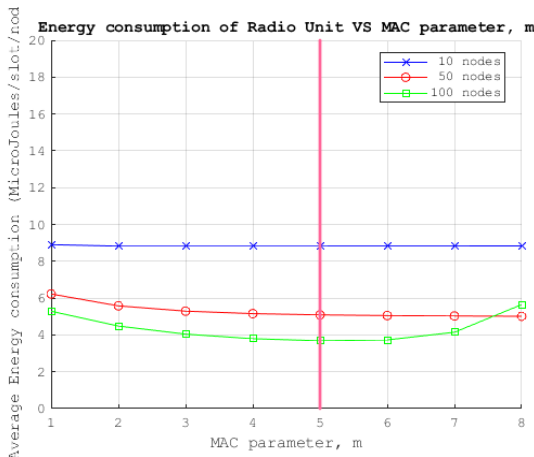

B

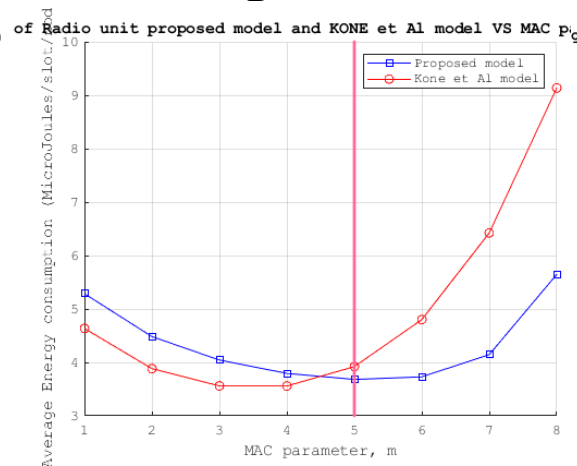

E

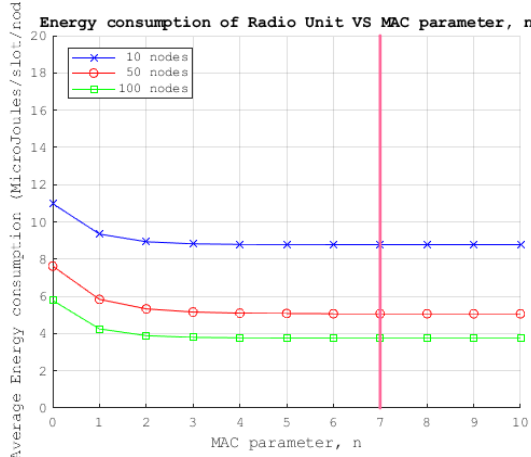

C

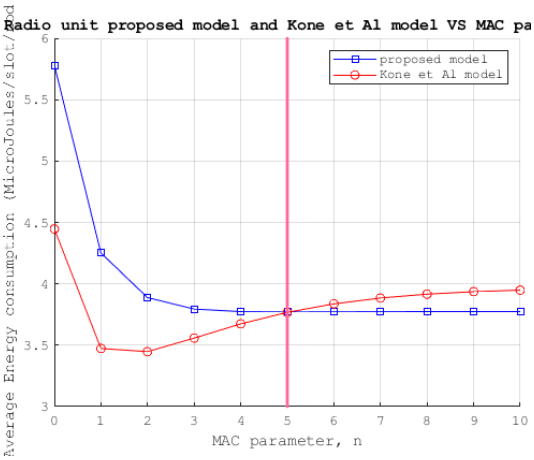

$\mathrm{F}$

Fig. 8. Average energy consumption of node vs MAC parameter: $m 0=1 \ldots 8, m b=8, m=0 \ldots 10, n=0 . .10, N=10 ; 50$; 100: $(\mathrm{A}$ and $\mathrm{D}) \mathrm{m0}=2$ to $8, \mathrm{mb}=8, \mathrm{~m}=4, \mathrm{n}=3$; $(\mathrm{B}$ and $\mathrm{E}) \mathrm{m}=1 . .8, \mathrm{mo}=3, \mathrm{mb}=8, \mathrm{n}=3$ and $(\mathrm{C}$ and $\mathrm{F}) \mathrm{n}=0 . .10$, $\mathrm{mo}=3, \mathrm{mb}=8, \mathrm{~m}=4$. For $D, E$ and $F, N=100$.

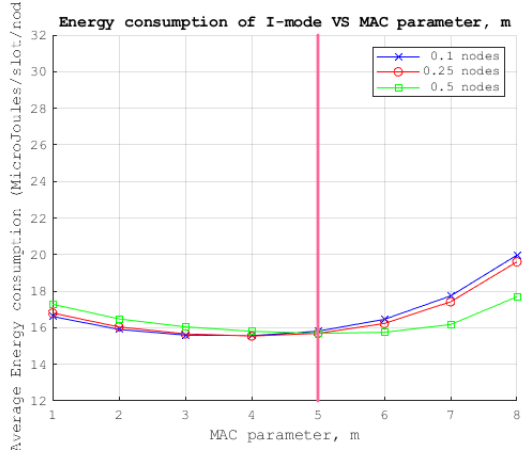

Fig.9. Average energy consumption of node $v s$ MAC parameter: $\mathrm{m}=1 . . .8, \mathrm{mo}=$ $3, \mathrm{mb}_{\mathrm{b}}=8, \mathrm{n}=3, \mathrm{~N}=100$ and $\mathrm{Ph}=$ $\{0.1,0.25,0.5\}$

\section{CONCLUSION}

In this paper, we have proposed and studied an enhanced version of the beacon-enabled slotted CSMA/CA 802.15.4 standard under the MAC parameters $m 0, m$ and $n$. We have

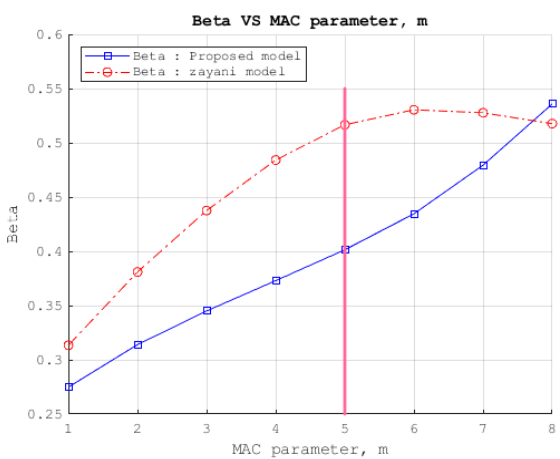

Fig.10 Busy channel probability during CCA 2 vs

MAC parameter: $\mathrm{m}=1 . . .8, \mathrm{m0}=$ $3, m_{b}=8, n=3, N=100$ and $P h=$ $\{0.5\}$

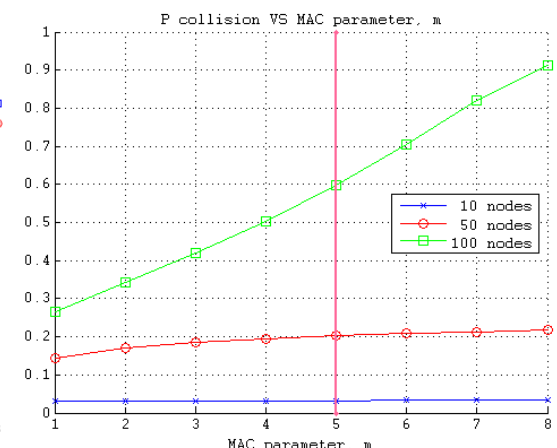

Fig.11 Collision probability vs MAC parameter: $\mathrm{m}=1 \ldots 8, \mathrm{mo}=3, \mathrm{mb}$ $=8, \mathrm{n}=3, \mathrm{~N}=100$ and $\mathrm{Ph} .=\{0.5\}$

analyzed the performance of following metrics: reliability, delay and average energy consumption of a node in 3 different star networks topologies.

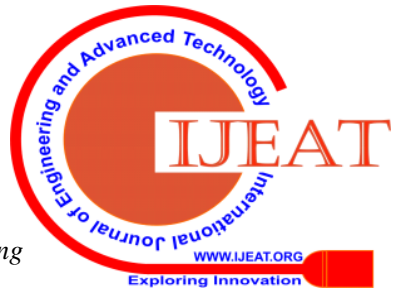


The numerical results show that our proposed model can improve significantly these metrics. Also, the results obtained indicate that the proposed model is more accurate than the original version proposed by park and enhanced by Zayani and Kone. It should be note that in general, $m_{0}$ is the main parameter which influence more the behaviour of node. But in some case one could associated the parameter $m$ or $n$ to $m_{0}$ in order to have a better performance.

\section{REFERENCES}

1. H. G. Diédié, M. Babri, et S. Oumtanaga, « Redundancy detection protocol for area coverage control in heterogeneous wireless sensor networks », Int. J. Comput. Sci. Issues, vol. 12, no 2, p. 11, 2015.

2. P. Park, P. Di Marco, C. Fischione, et K. H. Johansson, « Modeling and Optimization of the IEEE 802.15.4 Protocol for Reliable and Timely Communications », IEEE Trans. Parallel Distrib. Syst., vol. 24, no 3, p. 550-564, mars 2013, doi: 10.1109/TPDS.2012.159.

3. Y. Wang, W. Yang, R. Han, et K. You, « A Network Equivalent-Based Algorithm for Adaptive Parameter Tuning in 802.15.4 WSNs ", Sensors, vol. 18, no 7, p. 2031, juin 2018, doi: 10.3390/s18072031.

4. M. Nekrasov, R. Allen, I. Artamonova, et E. Belding, « Optimizing 802.15.4 Outdoor IoT Sensor Networks for Aerial Data Collection », Sensors, vol. 19, no 16, p. 3479, août 2019, doi: 10.3390/s19163479.

5. H. Alshaheen et H. Takruri-Rizk, « Energy Saving and Reliability for Wireless Body Sensor Networks (WBSN) », IEEE Access, vol. 6, p. 16678-16695, 2018, doi: 10.1109/ACCESS.2018.2817025.

6. K. Son, S. Hong, S.-P. Moon, T. Chang, et H. Cho, « Segmentized Clear Channel Assessment for IEEE 802.15.4 Networks », Sensors, vol. 16, no 6, p. 815, juin 2016, doi: 10.3390/s16060815.

7. P. Park, P. Di Marco, P. Soldati, C. Fischione, et K. H. Johansson, « A generalized Markov chain model for effective analysis of slotted IEEE 802.15.4 », in 2009 IEEE 6th International Conference on Mobile Adhoc and Sensor Systems, Macau, China, 2009, p. 130-139, doi: 10.1109/MOBHOC.2009.5337007.

8. M.-H. Zayani, V. Gauthier, et D. Zeghlache, « A Joint Model for IEEE 802.15.4 Physical and Medium Access Control Layers », ArXiv12053305 Cs, mai 2012.

9. C. T. Kone, A. Hafid, et M. Boushaba, « Performance Management of IEEE 802.15.4 Wireless Sensor Network for Precision Agriculture », IEEE Sens. J., vol. 15, no 10, p. 5734-5747, oct. 2015, doi: 10.1109/JSEN.2015.2442259.

10. G. Sharma, « Impact of BO and SO on Beacon-Enabled IEEE 802.15.4 », Int. J. Comput. Appl., vol. 66, p. 5.

11. A. K. Yadav, " MAC Layer Evaluation using IEEE 802.15.4 », Int. J. Res. Appl. Sci. Eng. Technol., vol. 7, no 11, p. 87-91, nov. 2019, doi: 10.22214/ijraset.2019.11016.

12. A. Sheraz et al., « Impact of Beacon Order and Superframe Order on IEEE 802.15.4 for Nodes Association in WBAN », EAI Endorsed Trans. Energy Web, vol. 5, no 17, p. 154454, avr. 2018, doi: 10.4108/eai.10-4-2018.154454.

13. A. F. Khalifeh, M. AlQudah, et K. A. Darabkh, « Optimizing the Beacon and SuperFrame orders in IEEE 802.15.4 for real-time notification in wireless sensor networks », in 2017 International Conference on Wireless Communications, Signal Processing and Networking (WiSPNET), Chennai, 2017, p. 595-598, doi: 10.1109/WiSPNET.2017.8299827.

14. H. Ayadi, A. Zouinkhi, B. Boussaid, M. N. Abdelkrim, T. Val, et S. Khssibi, " An effective approach for energy aware in wireless sensor network », in 2017 International Conference on Smart, Monitored and Controlled Cities (SM2C), Sfax, Tunisia, 2017, p. 128-133, doi: 10.1109/SM2C.2017.8071833.

15. Z. Xiao, J. Zhou, J. Yan, C. He, L. Jiang, et N. Trigoni, « Performance evaluation of IEEE 802.15.4 with real time queueing analysis », Ad Hoc Netw., vol. 73, p. 80-94, mai 2018, doi: 10.1016/j.adhoc.2018.01.006.

16. K. Katayama et H. Ohsaki, « Fluid-Based Modeling of Large-Scale IEEE 802.15.4 Wireless Sensor Networks », in 2019 IEEE 43rd Annual Computer Software and Applications Conference (COMPSAC), Milwaukee, WI, USA, 2019, p. 543-548, doi: 10.1109/COMPSAC.2019.10263.

17. M. Di Francesco, G. Anastasi, M. Conti, S. K. Das, et V. Neri, « Reliability and Energy-Efficiency in IEEE 802.15.4/ZigBee Sensor Networks: An Adaptive and Cross-Layer Approach », IEEE J. Sel. Areas Commun., vol. 29, no 8, p. 1508-1524, sept. 2011, doi: 10.1109/JSAC.2011.110902.

18. Yu-Chee Tseng, Sze-Yao Ni, et En-Yu Shih, « Adaptive approaches to relieving broadcast storms in a wireless multihop mobile ad hoc network », in Proceedings 21st International Conference on Distributed
Computing Systems, Mesa, AZ, USA, 2001, p. 481-488, doi: 10.1109/ICDSC.2001.918978.

19. H.-W. Tseng, Y.-C. Fan, S.-T. Sheu, et S.-Y. Ou, « An effective grouping scheme for avoiding hidden node problem in IEEE 802.15.4-based wireless sensor networks ", ACM SIGAPP Appl. Comput. Rev., vol. 14, no 1, p. 30-40, mars 2014, doi: 10.1145/2600617.2600620.

20. T. Elshabrawy, E. Shereen, M. Ashour, et J. Robert, « Report Success Probability/Battery Liftime Analysis of Dense IEEE 802.15.4-Based Metering Networks With Hidden Nodes », IEEE Sens. J., vol. 17, no 7, p. 2259-2266, avr. 2017, doi: 10.1109/JSEN.2017.2661879.

21. S. Ray, J. B. Carruthers, et D. Starobinski, " RTS/CTS-induced congestion in ad hoc wireless LANs ", in 2003 IEEE Wireless Communications and Networking, 2003. WCNC 2003., New Orleans, LA, USA, 2003, vol. 3, p. 1516-1521, doi: 10.1109/WCNC.2003.1200611.

22. R. Kaur et R. Sharma, " An Implementation and Analysis of RTS/CTS Mechanism for Data Transfer in Wireless Network », p. 6.

23. P. Sahoo, S. Pattanaik, et S.-L. Wu, «A Reliable Data Transmission Model for IEEE 802.15.4e Enabled Wireless Sensor Network under WiFi Interference », Sensors, vol. 17, no 6, p. 1320, juin 2017, doi: 10.3390/s17061320.

24. P. K. Sahoo, S. R. Pattanaik, et S.-L. Wu, « A Novel Synchronous MAC Protocol for Wireless Sensor Networks with Performance Analysis ", Sensors, vol. 19, no 24, p. 5394, déc. 2019, doi: 10.3390/s19245394.

25. E. Yundra, « Study of Adjustment Delay Scheme on IEEE 802.15.4 Networks at Beacon Enabled Mode », IOP Conf. Ser. Mater. Sci. Eng., vol. 288, p. 012065, janv. 2018, doi: 10.1088/1757-899X/288/1/012065

26. C. T. Kone, G. D. Sousa, et J. D. Mathias, " Energy-network performance trade-off in a slotted IEEE 802.15.4 network with acknowledged uplink transmissions », Int. J. Sens. Netw., vol. 17, no 4, p. 263, 2015, doi: 10.1504/IJSNET.2015.069581.

27. S. R. Pattanaik, P. K. Sahoo, et S.-L. Wu, « Performance Analysis of Modified IEEE 802.15.4e MAC for Wireless Sensor Networks », in Proceedings of the 14th ACM Symposium on Performance Evaluation of Wireless Ad Hoc, Sensor, \& Ubiquitous Networks - PE-WASUN '17, Miami, Florida, USA, 2017, p. 25-31, doi: 10.1145/3134829.3134833.

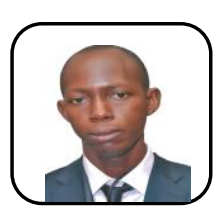

\section{AUTHORS PROFILE}

Karim Sidibe, received the master's degree in Computer Engineering from the University Nangui Abrogoua (UNA)/Ivory Coast and he is currently a PhD student at UNA. He is member of Laboratory of Mathmatic and Informatic of Fundamental and applied Sciences department. His current research interest is related to designing, modeling and optimizing Sensors

networks.

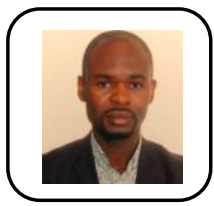

Dr Cheick Tidjane Kone, Cheick Tidjane Kone received the master's degree in network engineering and the Ph.D. degree in automatic control and computer engineering from Lorraine University, Nancy 1, France, in 2007 and 2011, respectively. He is currently an Assistant Professor with the École Supérieure Africaine des TICs, Ivory Coast. His current research interests include design, modeling, and performance evaluation of wireless sensor networks

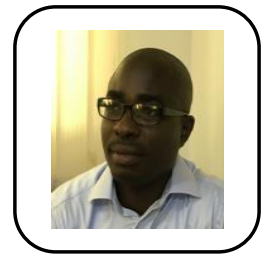

Dr Tchimou N'takpe, received the MS degree in engineering from Ecole Nationale d'Electricité et de Mécanique, Nancy, France, in 2005, and the PhD degree from Nancy University, France, in 2009 within the AlGorille INRIA project team. He is currently an assistant professor at the University Nangui Abrogoua, Abidjan, Côte d'Ivoire. His research interests include scheduling of parallel applications, optical networks, wireless sensor networks and internet of things. 
Wireless Sensor Node Performance Analysis Under Mac Protocol IEEE 802.15.4

Pr Aka Boko, head of computer training at Nangui Abrogoua University. He received PhD degree in 1989 from Louis Pasteur University in Strasbourg, France. He is a full professor at university Nangui Abrogoua (UNA)/Abidjan/Ivory Coast and he is specialized in computer networks and satellite data transmission. Member of mathematic and informatic laboratory of department of fundamental and applied sciences of UNA and director of the institute of research on renewable energies Ivory Coast. Currently his research fields cover computer sciences and network, data transmission, renewable energies.

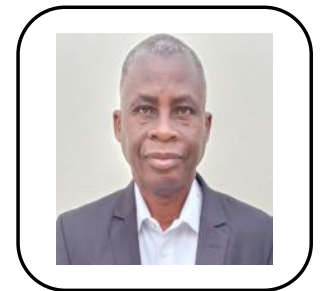

Pr Michel Babri, obtained his $\mathrm{PhD}$ from the University of Clermont-Ferrand II (France) in 1995. The scope of study was distributed applications. He is a full professor at National Polytechnic Institute Félix Houpouët-Boigny of Yamoussoukro (Côte d'Ivoire). Also he is the current Director of the Computer and Telecommunications Research Laboratory. His current research is in the field of optical networks and wireless sensor networks. He supervised several PhD theses: in WDM network; - elastic network; and in WSN. . In addition, I have been member or president of several PhD juries in Côte d'Ivoire and abroad. And he is supervising three on going $\mathrm{PhD}$ theses. 